\title{
Development of a Single-channel Multiple Immunoassay Chip
}

\author{
Masato TANAKA ${ }^{* 1}$, Toshihiko OOIE ${ }^{* 1}$, Yuji YAMACHOSHI ${ }^{* 1}$, Tomonori NAKAHARA ${ }^{* 1}$, \\ Mami HINO*2, Rie AKAMINE ${ }^{* 1}$, Masatoshi KATAOKA*1 \\ ${ }^{* 1}$ Health Technology Research Center, AIST, \\ Shikoku Center, 2217-14 Hayashi-cho Takamatsu, Kagawa 761-0395, Japan \\ E-mail: mst-tanaka@aist.go.jp \\ *2 College of Pharmaceutical Sciences, Matsuyama University, \\ 4-2 Bunkyo-cho, Matsuyama, Ehime 790-8578, Japan
}

\begin{abstract}
A novel micro immunoassay chip for detecting biomarkers in blood plasma has been developed. Since multiple detectors were made in a single channel, the chip was able to detect various biomarkers simultaneously. UV Laser processing of the substrate was performed to enhance immobilization of the antibody. This simple plastic chip requires only 1 micro liter per lane of blood plasma and 30 min of reaction time, one twentieth the sample volume required for the ELISA-kit and six times the through put.

DOI: $10.2961 / \mathrm{j} l \mathrm{mn} .2010 .01 .0008$
\end{abstract}

Keywords: microfluidic, excimer laser, inkjet, PMMA, COC, biomarker, antibody, antigen

\section{Introduction}

The Enzyme-Linked ImmunoSorbent Assay (ELISA) method is a method of detecting a small amount of albumin by measuring the amount of reaction after mixing an antibody and a measurement sample in wells on a plate. The ELISA method requires a sample size of $20 \mathrm{ml}$ or more. The antigen-antibody reaction takes at least three hours. A reduction in the sample quantity and shortening of the reactive time are needed for Point of Care Testing (POCT) [1]. We have developed a chip that allows for the antigenantibody reaction in a microscopic region in the channels. The chip uses an inkjet method to immobilize the antibody and laser surface treatment is applied.

\section{Experimental}

The immunoassay chip was produced using substrates processed under two irradiance conditions, untreated, laser processed. The antigen detection experiment was done, and the performances were compared. Additionally, a polymer coated substrate available on the market was used for the COC substrate.

\subsection{Laser Treatment of Substrates}

The experiment was conducted using two substrates, one of cycloolefin copolymer (COC) and another of polymethyl methacrylate (PMMA) [2-3].

Fig. 1 shows a schema of the substrate for the micro immunoassay chip. The substrate was made by injection molding. There were four channels of $300 \mu \mathrm{m}$ in width, $300 \mu \mathrm{m}$ in depth and $60 \mathrm{~mm}$ in length. Reagents for antigen-antibody reaction and chemiluminescence were injected from a sample inlet and exhausted from a sample outlet. An ArF excimar laser irradiated the part shown in light blue in Fig. 1, and the antibody dots were formed by the method described below.

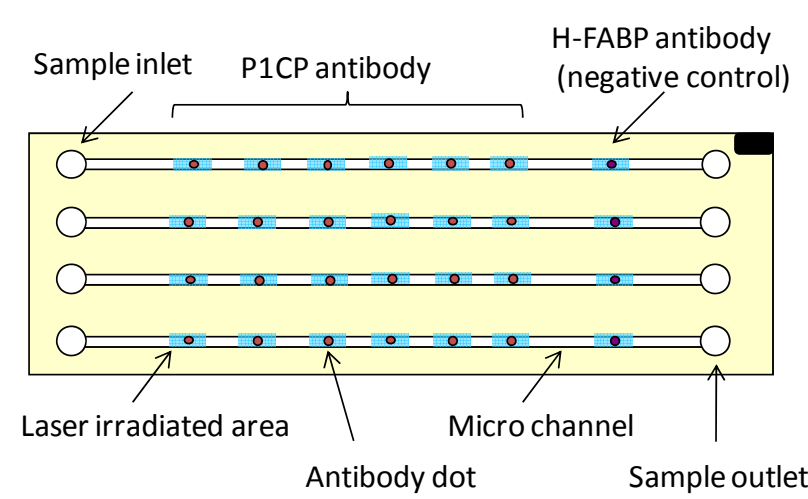

Fig. 1 Schematic illustration of a multipointimmunoassay chip with laser irradiated area for antibody immobilization.

Table 1 Specifications of the laser.

\begin{tabular}{|l|l|}
\hline Max. pulse energy & $500 \mathrm{~mJ}$ \\
\hline Max. power & $25 \mathrm{~W}$ \\
\hline Max. repetition rate & $50 \mathrm{pps}$ \\
\hline Pulse width & $14 \mathrm{~ns}$ \\
\hline
\end{tabular}

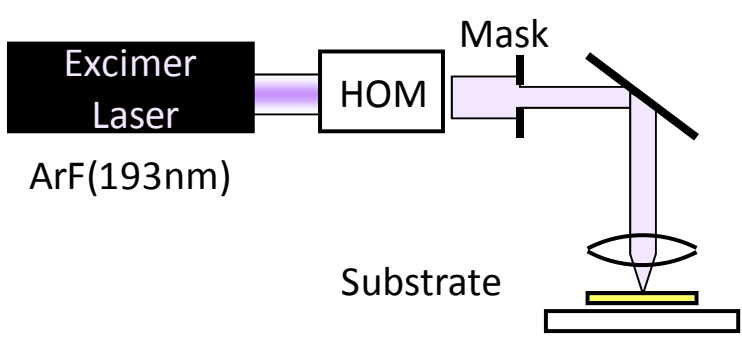

Fig. 2 Schematic diagram of the laser and optics used for the experiment. 
An ArF excimer laser (193 nm), LPX-305i (Coherent Inc.), was used for processing. The specifications of the laser oscillator are shown in Table 1. The laser processing system for this study is shown schematically in Fig. 2. The magnification was $1 / 10$ and the mask was oblong with apertures of $4.5 \times 10 \mathrm{~mm}$.

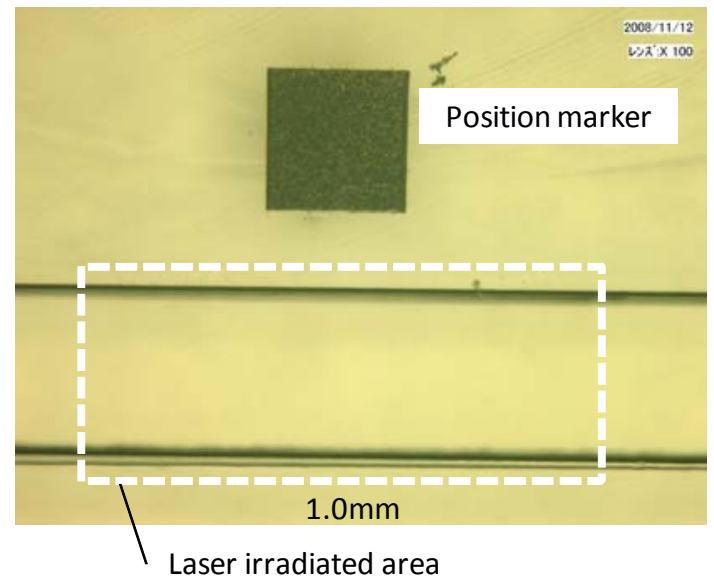

Fig. 3 An optical microscopic image of the laser irradiated area.

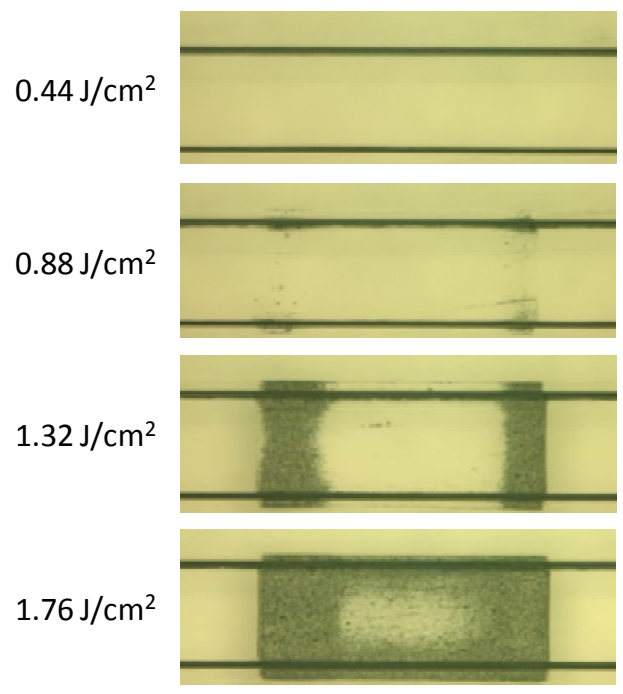

Fig. 4 Optical microscopic images of the laser irradiated area at various fluence.

Fig. 3 shows where the laser irradiated the channel bottom. A square mark near the channel is a marker to show where the antibody is fixed.

There was no change on externals in the irradiated area at the fluence of $0.44 \mathrm{~J} / \mathrm{cm}^{2}$. However, the transparency of the irradiated area decreased at $1.76 \mathrm{~J} / \mathrm{cm}^{2}$ as shown in Fig.4.

After a one minute wash with purified water using a sonicator the substrate was dried.

\subsection{Antibody Immobilization}

Procollagen type I c-peptide (P1CP) [4-5] and heart type fatty acid-binding protein (H-FABP) antibody solutions were ejected by an inkjet head onto the bottom surface of the channel. P1CP is used as one of the bone metabolism markers and H-FABP is a negative control here.

Fig. 5 shows a P1CP antibody solution in a micro channel. The volume of the ejected solution droplet was $113 \mathrm{pl}$, and 50 drops of product totaled $5.7 \mathrm{nl}$. There was no difference in the externals of the liquid drop when fixed on either the untreated areas or the laser-irradiated areas. The grooves were covered by a PMMA film with an adhesive layer to form micro channels.

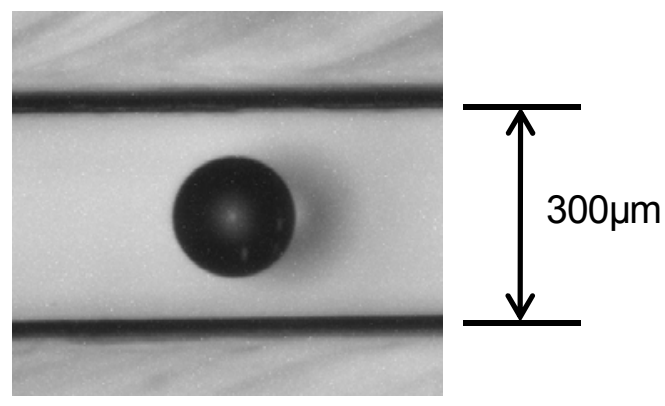

Fig. 5 Photograph of P1CP antibody solution in a micro channel ejected by an inkjet head driven by a piezoelectric actuator.

\subsection{Chemiluminescence Measurement}

The antigen detection operation with the chip is as follows. (1) Leave the chip at room temperature for four hours after the antibody solution is ejected.

(2) Fill the channels with the blocking liquid and leave for one hour.

(3) Inject and exhaust the cleaning solution three times.

(4) Fill the channels with the compound liquid of the P1CP antigen and the second antibody labeled with a peroxidase and leave for $30 \mathrm{~min}$.

(5) Inject and exhaust the cleaning solution five times.

(6) Fill the channels with a chemiluminescence substrate.

(7) Measure the signal intensity with a cooled CCD camera.

\section{Results and Discussion}

The results of the detection experiments of the P1CP antigen with the chips made from COC and PMMA are described. In addition, the results of multiple antigens detection are described.

\subsection{PICP antigen detection}

Fig. 6 shows the chemiluminescence when the P1CP solution of $640 \mathrm{ng} / \mathrm{ml}$ was used in the untreated, laser processed, and polymer coated COC substrates. Luminescence was observed in all the P1CP antibody dots. No luminescence was observed in the H-FABP antibody dots.

Fig. 7 is the comparison of the luminous intensities of the three substrates. The laser processed substrate, showed a nearly $30 \%$ increase in intensity from the untreated substrate. Also, the difference between dots decreased. A performance almost equal to the polymer coating was obtained. 


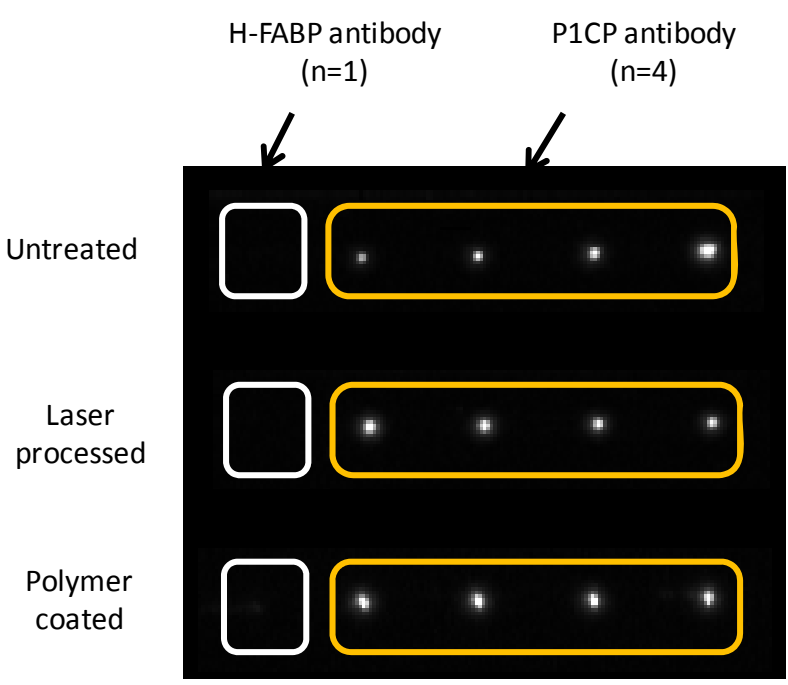

Fig. 6 An image of chemiluminescence from detection dots with a P1CP antigen solution of $640 \mathrm{ng} / \mathrm{ml}$ in density. Exposure time was $20 \mathrm{~min}$.

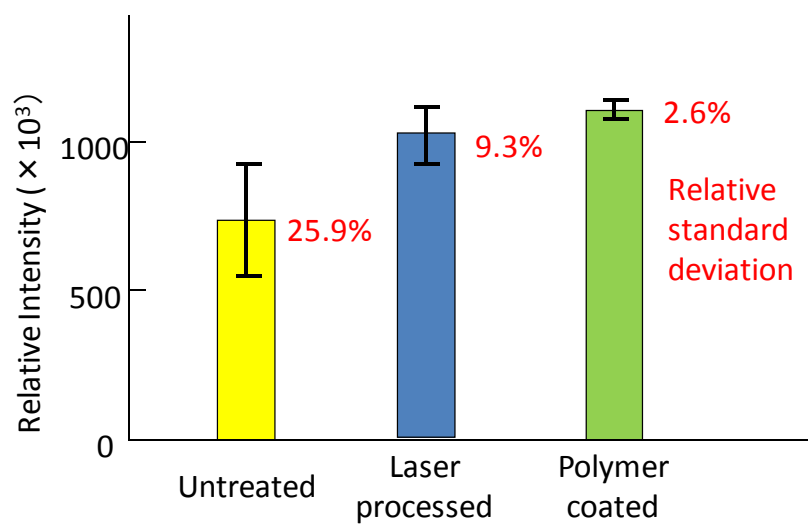

Fig. 7 Comparison of relative signal intensity and relative standard deviation of each chip.

Fig. 8 shows the change in the signal intensity according to the laser fluence. When the fluence increased, the signal intensity increased and the dispersion between the detection dots decreased.

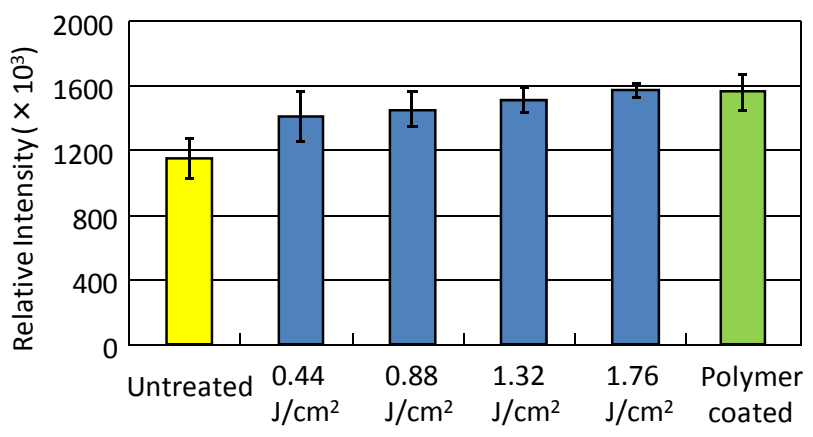

Fig. 8 Comparison of relative signal intensity and relative standard deviation of each chip.
Next, the performance results of tests to measure the antigen concentration using the microchip are described. Fig. 9 shows the chemiluminescence with the P1CP antigen at concentrations of $0,150,300$, and $600 \mathrm{ng} / \mathrm{ml}$.

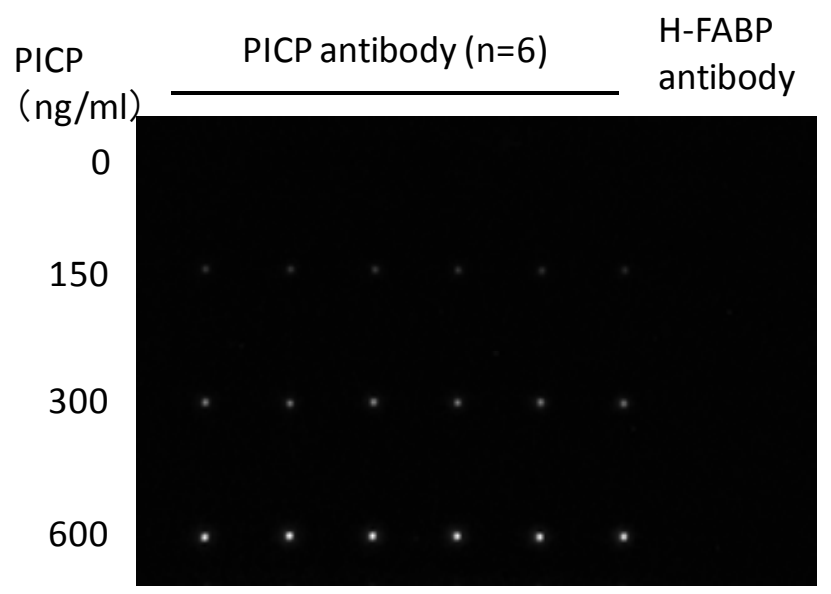

Fig. 9 An image of chemiluminescence from detection dots with P1CP antigen solutions of $0,150,300$, and $600 \mathrm{ng} / \mathrm{ml}$ in density. Exposure time was $15 \mathrm{~min}$.

Fig. 10 shows the intensity of each detection dot from Fig. 9. This graph indicates that the signal intensity and the antigen concentration had high linearity. The detection limit of this assay was $5 \mathrm{ng} / \mathrm{ml}$ and it was a little lower than the ELISA method.

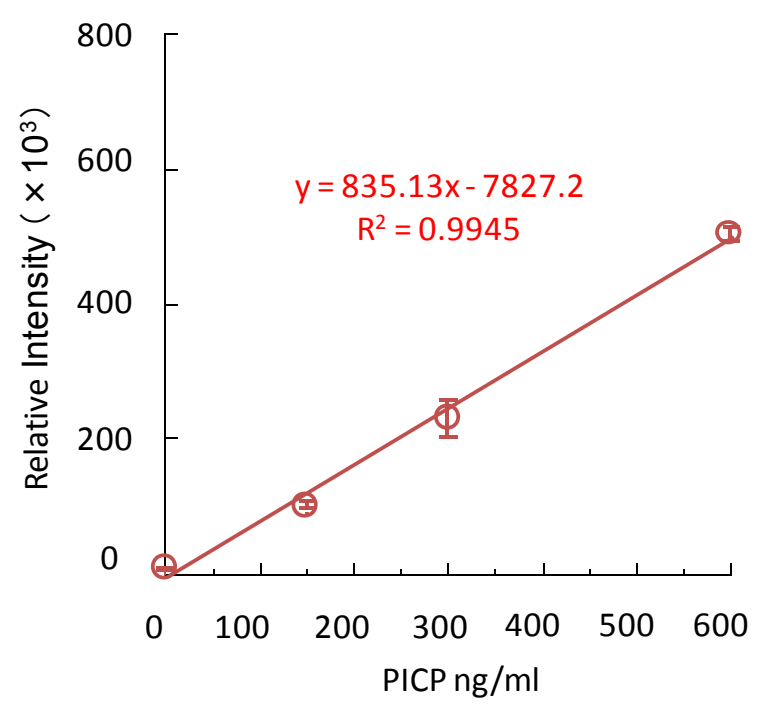

Fig. 10 The relationship between the signal intensity and the concentration of P1CP antigen.

\subsection{The PMMA substrate}

Injection molded substrates made of PMMA with a micro groove were prepared. The manufacturing method of the chips was similar to the case of the COC substrate. However, the width $(500 \mu \mathrm{m})$ and length differed. 
Untreated and laser processed PMMA substrates and a polymer coated COC substrate were tested. The laser processing was done with a fluence of $0.32 \mathrm{~J} / \mathrm{cm}^{2}$. The grooves were covered by a PMMA film with an adhesive layer to form micro channels. P1CP antibody solutions of $5.3 \mathrm{nl}$ were ejected at each detection dot.

A P1CP antigen solution with a concentration of 640 $\mathrm{ng} / \mathrm{ml}$ was used on the chips. The results of the detection performance are shown in Fig. 11. It shows that the signal intensity increased remarkably due to laser irradiation.

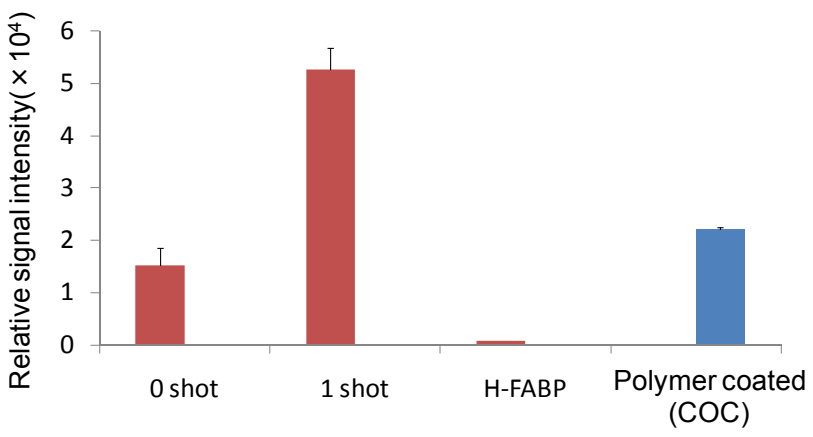

Fig. 11 Comparison of relative signal intensity and relative standard deviation of each chip made from PMMA.

\subsection{Multiple Immunoassay}

An example using three kinds of antibody in one channel is shown as follows. The COC polymer coated substrate was used here. The antibodies used were interleukin6 (IL-6), tumor necrosis factor $\alpha$ (TNF $\alpha$ ), and adiponectin.

Fig. 12 shows the result of measuring an actual blood plasma sample. With this chip, it was shown that the simultaneous detection of two or more antigens was possible from seeing distinct luminescence in all dots.

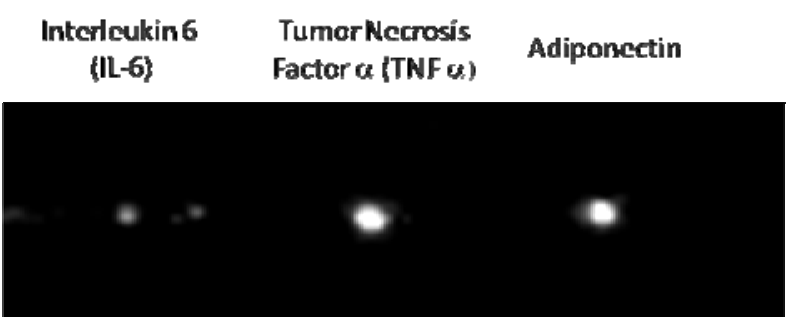

Fig. 12 An image of chemiluminescence from detection dots when three kinds of antigens were detected simultaneously. Exposure time was $30 \mathrm{~min}$.

\section{Conclusions}

A micro immunoassay chip for detecting biomarkers in blood plasma has been developed. The chip requires only 1 micro liter per lane of sample and 30 minutes reaction time. Antibody immobilization was an important factor in the performance of the chip. Tests showed that laser surface processing is effective for increasing signal intensity and reducing relative standard deviation in signal intensity.

\section{References}

[1] E. Eteshola, D. Leckband: Sensors and Acutuators BChenmilal, 72, (2001) 129

[2] J. Brandrup and E. H. Immergut, Polymer Handbook $3^{\text {rd }}$ ed. ISBN 0-471-812447-7, V/77

[3] B. Crist, M. E. Marhic, G. Raviv and M. Epstein, J. Appl. Phys., 51(2), (1980) 1160

[4] Taubman, M.B., Goldberg, B., Sherr, C.J.: Radioimmunoassay for human procollagen, Sceinece, 186, (1974) 1115

[5] Savolainen, E.R., Goldberg, B., Leo, M.A., et al., Alcoholism: Chemical and Experimental Research, 8, (1984) 384 